MATHEMATICS OF COMPUTATION

Volume 72, Number 242, Pages 767-786

S 0025-5718(02)01451-5

Article electronically published on June 4, 2002

\title{
NEAR OPTIMALITY OF THE SINC APPROXIMATION
}

\author{
MASAAKI SUGIHARA
}

\begin{abstract}
Near optimality of the sinc approximation is established in a variety of spaces of functions analytic in a strip region about the real axis, each space being characterized by the decay rate of their elements (functions) in the neighborhood of the infinity.
\end{abstract}

\section{INTRODUCTION}

Let $f$ be a function defined on the real line $\mathbf{R}$. The $N(=2 n+1)$-point sinc approximation is defined by

$$
f(x) \approx \sum_{j=-n}^{n} f(j h) S(j, h)(x),
$$

where $S(j, h)(x)$ is the so-called sinc function defined by

$$
S(j, h)(x)=\frac{\sin [(\pi / h)(x-j h)]}{(\pi / h)(x-j h)},
$$

and the step size $h$ is suitably chosen for a given positive integer $n$.

In the area of numerical analysis the use of the sinc approximation has been extensively studied during the last two decades. A variety of highly accurate approximation procedures have been derived for interpolating, integrating, evaluating the Fourier and the Hilbert transforms of functions, and solving the ordinary and the partial differential equations. These approximation procedures are now collectively referred to as sinc numerical methods (see Kowalski, Sikorski and Stenger [9], Lund and Bowers [10, and Stenger [17, 18]).

While the use of the sinc approximation was being studied, a great advance was made also in the theory of the sinc approximation. That is to say, the near optimality of the sinc approximation was established. To state the result precisely, we need the following definition.

Definition 1.1. (1) For a positive number $d, \mathcal{D}_{d}$ denotes the strip region of width $2 d$ about the real axis:

$$
\mathcal{D}_{d}=\{z \in \mathbf{C}|| \operatorname{Im} z \mid<d\}
$$

Received by the editor July 10, 2000 and, in revised form, August 27, 2001.

2000 Mathematics Subject Classification. Primary 41A30, 41A25, 65D15.

Key words and phrases. Sinc approximation, near optimality, variable transformation, double exponential formula.

The author was supported in part by a Grant-in-Aid for Scientific Research from the Ministry of Education, Sports, Culture and Science, and by the Japan Society for Promotion of Science.

(C)2002 American Mathematical Society 
(2) For $1<p \leq \infty$, putting

$$
\mathcal{N}_{p}^{*}(f, y) \equiv\left\{\begin{array}{rr}
\left(\int_{-\infty}^{+\infty}|f(x+i y)|^{p}|\cosh (x+i y)|^{2 p / q}\right. \\
\left.\quad+|f(x-i y)|^{p}|\cosh (x-i y)|^{2 p / q} d x\right)^{1 / p} & \text { for } 1<p<\infty, \\
\sup _{z \in \mathcal{D}_{y}}\left|f(z) \cosh ^{2}(z)\right| & \text { for } p=\infty,
\end{array}\right.
$$

where $1 / p+1 / q=1$, we define $\boldsymbol{H}_{p}^{*}\left(\mathcal{D}_{\pi / 4}\right)$ to be the following:

$$
\begin{aligned}
\boldsymbol{H}_{p}^{*}\left(\mathcal{D}_{\pi / 4}\right)=\left\{f: \mathcal{D}_{\pi / 4} \rightarrow \mathbf{C} \mid\right. & f(z) \text { is analytic in } \mathcal{D}_{\pi / 4}, \\
& \mathcal{N}_{p}^{*}(f, y)<\infty \text { for all } y \text { with } 0 \leq y<\pi / 4, \\
& \text { and } \left.\|f\|_{p}^{*} \equiv \lim _{y \rightarrow \pi / 4-0} \mathcal{N}_{p}^{*}(f, y)<\infty\right\}
\end{aligned}
$$

(3) For the $N(=2 n+1)$-point sinc approximation formula, $E_{N, h}^{\operatorname{sinc}}\left(\boldsymbol{H}_{p}^{*}\left(\mathcal{D}_{\pi / 4}\right)\right)$ denotes the error norm in $\boldsymbol{H}_{p}^{*}\left(\mathcal{D}_{\pi / 4}\right)$ :

$$
E_{N, h}^{\mathrm{sinc}}\left(\boldsymbol{H}_{p}^{*}\left(\mathcal{D}_{\pi / 4}\right)\right)=\sup _{\|f\|_{p}^{*} \leq 1}\left\{\sup _{x \in \mathbf{R}}\left|f(x)-\sum_{j=-n}^{n} f(j h) S(j, h)(x)\right|\right\},
$$

and $E_{N}^{\min }\left(\boldsymbol{H}_{p}^{*}\left(\mathcal{D}_{\pi / 4}\right)\right)$ designates the minimum error norm in $\boldsymbol{H}_{p}^{*}\left(\mathcal{D}_{\pi / 4}\right)$, where the minimum is taken over all the $N$-point approximation formulas:

$E_{N}^{\min }\left(\boldsymbol{H}_{p}^{*}\left(\mathcal{D}_{\pi / 4}\right)\right)$

$$
=\inf _{1 \leq l \leq N} \inf _{\substack{m_{1}, m_{2}, \ldots, m_{l} \\ m_{1}+m_{2}+\cdots+m_{l}=N}} \inf _{a_{j} \in \mathcal{D}_{\pi / 4}} \inf _{\text {distinct }}\left\{\sup _{\phi_{j k}}\left\{\sup _{\|f\|_{p}^{*} \leq 1}\left|f(x)-\sum_{j=1}^{l} \sum_{k=0}^{m_{j}-1} f^{(k)}\left(a_{j}\right) \phi_{j k}(x)\right|\right\}\right\},
$$

where $\phi_{j k}$ 's are functions analytic in $\mathcal{D}_{\pi / 4}$. It should be clear that $a_{j}$ 's represent the node points, $\phi_{j k}$ 's designate the basis functions, and $m_{j}$ 's are the maximal orders of the derivatives.

The optimality result, claiming that $E_{N}^{\min }\left(\boldsymbol{H}_{p}^{*}\left(\mathcal{D}_{\pi / 4}\right)\right) \approx E_{N, h}^{\operatorname{sinc}}\left(\boldsymbol{H}_{p}^{*}\left(\mathcal{D}_{\pi / 4}\right)\right)$, reads as follows:

Theorem 1.1 ([4, 14, 21]). (1) For $1<p<\infty$, there exist constants $C_{p}, C_{p}^{\prime}$ such that

$$
\begin{aligned}
C_{p} N^{-1 / 2 p} \exp \left(-\pi \sqrt{\frac{N}{2 q}}\right) & \leq E_{N}^{\min }\left(\boldsymbol{H}_{p}^{*}\left(\mathcal{D}_{\pi / 4}\right)\right) \\
& \leq E_{N, h}^{\operatorname{sinc}}\left(\boldsymbol{H}_{p}^{*}\left(\mathcal{D}_{\pi / 4}\right)\right) \leq C_{p}^{\prime} \sqrt{N} \exp \left(-\frac{\pi}{2} \sqrt{\frac{N}{q}}\right),
\end{aligned}
$$

where $q=p /(p-1), N=2 n+1$ and the step size $h$ of the sinc approximation is chosen as $h=(\pi / 2) \sqrt{q /(2 n)}$.

(2) For $p=\infty$, there exist constants $C_{\infty}, C_{\infty}^{\prime}, \nu$ such that

$$
\begin{aligned}
C_{\infty} N^{\nu} \exp (-\pi \sqrt{N}) & \leq E_{N}^{\min }\left(\boldsymbol{H}_{\infty}^{*}\left(\mathcal{D}_{\pi / 4}\right)\right) \\
& \leq E_{N}^{\operatorname{sinc}}\left(\boldsymbol{H}_{\infty}^{*}\left(\mathcal{D}_{\pi / 4}\right)\right) \leq C_{\infty}^{\prime} \sqrt{N} \exp \left(-\frac{\pi}{2} \sqrt{N}\right),
\end{aligned}
$$


where $N=2 n+1$ and the step size $h$ of the sinc approximation is chosen as $h=(\pi / 2) \sqrt{1 /(2 n)}$.

The upper bounds for $E_{N}^{\operatorname{sinc}}\left(\boldsymbol{H}_{p}^{*}\left(\mathcal{D}_{\pi / 4}\right)\right)$ in (1) and for $E_{N}^{\operatorname{sinc}}\left(\boldsymbol{H}_{\infty}^{*}\left(\mathcal{D}_{\pi / 4}\right)\right)$ in $(2)$ are due to Stenger [14]. The lower bound for $E_{N}^{\min }\left(\boldsymbol{H}_{p}^{*}\left(\mathcal{D}_{\pi / 4}\right)\right)$ in (1) is due to Wilderotter [21, and the lower bound for $E_{N}^{\min }\left(\boldsymbol{H}_{\infty}^{*}\left(\mathcal{D}_{\pi / 4}\right)\right)$ in $(2)$ is due to Burchard and Höllig 4].

The space $\boldsymbol{H}_{p}^{*}\left(\mathcal{D}_{\pi / 4}\right)$ above may appear strange at first sight, but an examination of the definition shows that $\boldsymbol{H}_{p}^{*}\left(\mathcal{D}_{\pi / 4}\right)$ is nothing but the space of functions which are analytic in the strip region $\mathcal{D}_{\pi / 4}$ and which decay exponentially in the neighborhood of infinity. Hence the theorem above claims that the sinc approximation is nearly optimal in the space of functions which are analytic in the strip region $\mathcal{D}_{\pi / 4}$ and which decay exponentially in the neighborhood of infinity. In view of this the question naturally arises of whether or not the sinc approximation is still nearly optimal in other spaces, specifically, in the space of analytic functions enjoying other types of decay property.

The main aim of the present paper is to answer this question in the affirmative, that is, to show that the sinc approximation is nearly optimal in a variety of spaces of functions analytic in the strip region $\mathcal{D}_{d}$. Each of the spaces, denoted by $\boldsymbol{H}^{\infty}\left(\mathcal{D}_{d}, \omega\right)$, is characterized by the decay rate of their elements (functions), parameterized by $\omega$, in the neighborhood of infinity. The space $\boldsymbol{H}^{\infty}\left(\mathcal{D}_{d}, \omega\right)$ has recently been introduced by the author in establishing the "meta-optimality" of the double exponential quadrature formulas ([19]).

The paper is organized as follows: In Section 2 the spaces $\boldsymbol{H}^{\infty}\left(\mathcal{D}_{d}, \omega\right)$ are defined; in Section 3 we state the optimality results for the sinc approximation in $\boldsymbol{H}^{\infty}\left(\mathcal{D}_{d}, \omega\right)$; Section 4 affords the proofs of the optimality results; and in Section 5 several remarks are made.

\section{Function spaces $\boldsymbol{H}^{\infty}\left(\mathcal{D}_{d}, \omega\right)$}

We give the definition of the function space $\boldsymbol{H}^{\infty}\left(\mathcal{D}_{d}, \omega\right)$. The space represents the space of functions analytic in the strip region $\mathcal{D}_{d}$ about the real axis, being characterized by the decay rate of its elements (functions) in the neighborhood of infinity. This $\boldsymbol{H}^{\infty}\left(\mathcal{D}_{d}, \omega\right)$ has recently been introduced in the context of optimal quadrature in [19].

Definition 2.1. Let $\omega(z)$ be a function defined on the strip region $\mathcal{D}_{d}$ of width $2 d$ about the real axis, and let us assume that $\omega(z)$ does not vanish at any point of $\mathcal{D}_{d}$. Define the space $\boldsymbol{H}^{\infty}\left(\mathcal{D}_{d}, \omega\right)$ by

$$
\boldsymbol{H}^{\infty}\left(\mathcal{D}_{d}, \omega\right)=\left\{f: \mathcal{D}_{d} \rightarrow \mathbf{C} \mid f(z) \text { is analytic in } \mathcal{D}_{d}, \text { and }\|f\|<+\infty\right\},
$$

where the norm of $f$ is given by

$$
\|f\|=\sup _{z \in \mathcal{D}_{d}}|f(z) / \omega(z)| .
$$

From the definition of the norm, we know that

$$
|f(z)| \leq\|f\||\omega(z)| \quad\left(z \in \mathcal{D}_{d}\right)
$$

which means that $f \in \boldsymbol{H}^{\infty}\left(\mathcal{D}_{d}, \omega\right)$ decays like $\omega(z)$ as $z \rightarrow \infty$ in $\mathcal{D}_{d}$. For instance, for $\omega(z)$ that decays single exponentially the functions in $\boldsymbol{H}^{\infty}\left(\mathcal{D}_{d}, \omega\right)$ decay single exponentially, and for $\omega(z)$ that decays double exponentially the functions 
in $\boldsymbol{H}^{\infty}\left(\mathcal{D}_{d}, \omega\right)$ decay double exponentially. As examples of $\omega(z)$, we consider the following.

1. Single exponential decay type:

$$
\operatorname{sech}^{\mu}(z) \quad(\mu>0), \quad \exp \left(-z^{2}\right) .
$$

2. Double exponential decay type:

$$
\operatorname{sech}^{\mu}\left(\frac{\pi}{2} \sinh (z)\right) \quad(\mu>0), \quad \exp (-A \cosh (B z)) \quad(A, B>0) .
$$

\section{Statement of the optimality Results}

For the sinc approximation formula, let $E_{N, h}^{\operatorname{sinc}}\left(\boldsymbol{H}^{\infty}\left(\mathcal{D}_{d}, \omega\right)\right)$ denote the error norm in $\boldsymbol{H}^{\infty}\left(\mathcal{D}_{d}, \omega\right)$ :

$$
E_{N, h}^{\text {sinc }}\left(\boldsymbol{H}^{\infty}\left(\mathcal{D}_{d}, \omega\right)\right)=\sup _{\|f\| \leq 1}\left\{\sup _{x \in \mathbf{R}}\left|f(x)-\sum_{j=-n}^{n} f(j h) S(j, h)(x)\right|\right\},
$$

and let $E_{N}^{\min }\left(\boldsymbol{H}^{\infty}\left(\mathcal{D}_{d}, \omega\right)\right)$ designate the minimum error norm in $\boldsymbol{H}^{\infty}\left(\mathcal{D}_{d}, \omega\right)$, where the minimum is taken over all the $N$-point approximation formulas:

$$
\begin{aligned}
E_{N}^{\min }\left(\boldsymbol{H}^{\infty}\left(\mathcal{D}_{d}, \omega\right)\right) & \\
\quad= & \inf _{1 \leq l \leq N} \inf _{\substack{m_{1}, m_{2}, \ldots, m_{l}=N \\
m_{1}+m_{2}+\cdots+m_{l}=N}} \inf _{\substack{a_{j} \in \mathcal{D}_{d} \\
\text { distinct }}} \inf _{\phi_{j k}}\left\{\sup _{\|f\| \leq 1}\left\{\sup _{x \in \mathbf{R}}\left|f(x)-\sum_{j=1}^{l} \sum_{k=0}^{m_{j}-1} f^{(k)}\left(a_{j}\right) \phi_{j k}(x)\right|\right\}\right\},
\end{aligned}
$$

Following Stenger ([15, [17]), we denote by $B\left(\mathcal{D}_{d}\right)$ the family of all functions $f$ analytic in $\mathcal{D}_{d}$ such that

$$
\int_{-d}^{d}|f(x+i y)| d y \rightarrow 0 \quad \text { as } x \rightarrow \pm \infty
$$

and such that $\mathcal{N}_{1}\left(f, \mathcal{D}_{d}\right)<\infty$, where

$$
\mathcal{N}_{1}\left(f, \mathcal{D}_{d}\right) \equiv \lim _{y \rightarrow d-0} \int_{-\infty}^{+\infty}|f(x+i y)|+|f(x-i y)| d x .
$$

Just as in the theory of error analysis of the sinc approximation formula, this family $B\left(\mathcal{D}_{d}\right)$ plays an important role later in the technical developments in this paper.

The following two theorems give the optimality results on the sinc approximation in $\boldsymbol{H}^{\infty}\left(\mathcal{D}_{d}, \omega\right)$ : the first theorem is for the case where the decay rate of $\omega(z)$ is single exponential, which implies that the functions to be approximated decay single exponentially; and the second theorem is for the case of double exponential decay of $\omega(z)$. The theorems claim the near-optimality of the sinc approximation formula in $\boldsymbol{H}^{\infty}\left(\mathcal{D}_{d}, \omega\right)$ in the sense that, provided the mesh size $h$ of the sinc approximation formula is suitably chosen,

$$
E_{N, h}^{\operatorname{sinc}}\left(\boldsymbol{H}^{\infty}\left(\mathcal{D}_{d}, \omega\right)\right) \approx E_{N}^{\min }\left(\boldsymbol{H}^{\infty}\left(\mathcal{D}_{d}, \omega\right)\right) .
$$

The proofs of the theorems are given in the next section. 
Theorem 3.1. Suppose that the function $\omega(z)$ meets the following three conditions:

1. $\omega(z) \in B\left(\mathcal{D}_{d}\right)$;

2. $\omega(z)$ does not vanish at any point in $\mathcal{D}_{d}$;

3. the decay rate of $\omega(z)$ on the real axis satisfies

$$
\alpha_{1} \exp \left(-(\beta|x|)^{\rho}\right) \leq|\omega(x)| \leq \alpha_{2} \exp \left(-(\beta|x|)^{\rho}\right), \quad-\infty<x<\infty,
$$

where $\alpha_{1}, \alpha_{2}, \beta>0$, and $\rho \geq 1$.

Then

$$
E_{N, h}^{\mathrm{sinc}}\left(\boldsymbol{H}^{\infty}\left(\mathcal{D}_{d}, \omega\right)\right) \leq C_{d, \omega} N^{\frac{1}{\rho+1}} \exp \left(-\left(\frac{\pi d \beta N}{2}\right)^{\frac{\rho}{\rho+1}}\right)
$$

where $N=2 n+1$, the mesh size $h$ is chosen as

$$
h=(\pi d)^{\frac{1}{\rho+1}}(\beta n)^{-\frac{\rho}{\rho+1}}
$$

and $C_{d, \omega}$ is a constant depending on $d$ and $\omega$, and

$$
E_{N}^{\min }\left(\boldsymbol{H}^{\infty}\left(\mathcal{D}_{d}, \omega\right)\right) \geq C_{d, \omega}^{\prime} \exp \left(-\left(\left(\frac{2}{\rho+1}\right)^{\frac{1}{\rho}} \pi d \beta N\right)^{\frac{\rho}{\rho+1}}\right)
$$

where $C_{d, \omega}^{\prime}$ is another constant depending on $d$ and $\omega$.

Theorem 3.2. Suppose that the function $\omega(z)$ meets the following three conditions:

1. $\omega(z) \in B\left(\mathcal{D}_{d}\right)$;

2. $\omega(z)$ does not vanish at any point in $\mathcal{D}_{d}$;

3. the decay rate of $\omega(z)$ on the real axis satisfies

$\alpha_{1} \exp \left(-\beta_{1} \exp (\gamma|x|)\right) \leq|\omega(x)| \leq \alpha_{2} \exp \left(-\beta_{2} \exp (\gamma|x|)\right), \quad-\infty<x<\infty$, where $\alpha_{1}, \alpha_{2}, \beta_{1}, \beta_{2}, \gamma>0$.

Then

$$
E_{N, h}^{\mathrm{sinc}}\left(\boldsymbol{H}^{\infty}\left(\mathcal{D}_{d}, \omega\right)\right) \leq C_{d, \omega} \exp \left(-\frac{\pi d \gamma N}{2 \log \left(\pi d \gamma N /\left(2 \beta_{2}\right)\right)}\right),
$$

where $N=2 n+1$, the mesh size $h$ is chosen as

$$
h=\frac{\log \left(\pi d \gamma n / \beta_{2}\right)}{\gamma n}
$$

and $C_{d, \omega}$ is a constant depending on $d$ and $\omega$, and

$$
E_{N}^{\min }\left(\boldsymbol{H}^{\infty}\left(\mathcal{D}_{d}, \omega\right)\right) \geq C_{d, \omega}^{\prime} \exp \left(-\frac{\pi d \gamma N}{\log \left(\pi d \gamma N /\left(2 \beta_{1}\right)\right)}\right)
$$

where $C_{d, \omega}^{\prime}$ is another constant depending on $d$ and $\omega$.

Remark 3.1. It suffices to consider at most the case where the decay rate of $\omega(z)$ is double exponential. In fact the following theorem, established recently in [19], holds good. 
Theorem 3.3 ([19]). There exists no function $\omega(z)$ that satisfies the following three conditions:

1. $\omega(z) \in B\left(\mathcal{D}_{d}\right)$;

2. $\omega(z)$ does not vanish at any point in $\mathcal{D}_{d}$;

3. the decay rate of $\omega(z)$ on the real axis is specified as

$$
\omega(x)=\mathrm{O}(\exp (-\beta \exp (\gamma|x|))) \quad \text { as }|x| \rightarrow \infty,
$$

where $\beta>0$ and $\gamma>\pi /(2 d)$.

Examples. We consider the cases where $\omega(z)$ are given by (2.2) and (2.3).

(a) The function

$$
\omega(z)=\operatorname{sech}^{\mu}(z) \quad(\mu>0)
$$

satisfies all the conditions in Theorem 3.1 with $\beta=\mu$ and $\rho=1$, provided $d<\pi / 2$. (We here omit the values of $\alpha_{1}$ and $\alpha_{2}$, for they are irrelevant to the orders of magnitude of $E_{N, h}^{\text {sinc }}$ and $E_{N}^{\min }$. For the same reason we will do so in the following examples.) Hence Theorem 3.1 yields

$$
E_{N, h}^{\text {sinc }}\left(\boldsymbol{H}^{\infty}\left(\mathcal{D}_{d}, \omega\right)\right) \leq C_{d, \mu} \sqrt{N} \exp (-\sqrt{\pi d \mu N / 2}),
$$

where $N=2 n+1, h=\sqrt{\pi d /(\mu n)}$ and $C_{d, \mu}$ is a constant depending on $d$ and $\mu$, and

$$
E_{N}^{\min }\left(\boldsymbol{H}^{\infty}\left(\mathcal{D}_{d}, \omega\right)\right) \geq C_{d, \mu}^{\prime} \exp (-\sqrt{\pi d \mu N})
$$

where $C_{d, \mu}^{\prime}$ is another constant depending on $d$ and $\mu$.

(b) The function

$$
\omega(z)=\exp \left(-z^{2}\right)
$$

satisfies all the conditions in Theorem 3.1 with $\beta=1$ and $\rho=2$. Hence Theorem 3.1 yields

$$
E_{N, h}^{\text {sinc }}\left(\boldsymbol{H}^{\infty}\left(\mathcal{D}_{d}, \omega\right)\right) \leq C_{d} N^{1 / 3} \exp \left(-(\pi d N)^{2 / 3}\right),
$$

where $N=2 n+1, h=2(\pi d)^{1 / 3} n^{-2 / 3}$ and $C_{d}$ is a constant depending on $d$, and

$$
E_{N}^{\min }\left(\boldsymbol{H}^{\infty}\left(\mathcal{D}_{d}, \omega\right)\right) \geq C_{d}^{\prime} \exp \left(-\left(\left(\frac{2}{3}\right)^{1 / 2} \pi d N\right)^{2 / 3}\right)
$$

where $C_{d}^{\prime}$ is another constant depending on $d$.

(c) The function

$$
\omega(z)=\operatorname{sech}^{\mu}\left(\frac{\pi}{2} \sinh (z)\right) \quad(\mu>0)
$$

satisfies all the conditions in Theorem 3.2 with $\beta_{1}=\beta_{2}=\pi \mu / 4$ and $\gamma=1$, provided $d<\pi / 2$. Hence Theorem 3.2 yields

$$
E_{N, h}^{\text {sinc }}\left(\boldsymbol{H}^{\infty}\left(\mathcal{D}_{d}, \omega\right)\right) \leq C_{d, \mu} \exp \left(-\frac{\pi d N}{2 \log (2 d N / \mu)}\right),
$$

where $N=2 n+1, h=\log (4 d n / \mu) / n$ and $C_{d, \mu}$ is a constant depending on $d$ and $\mu$, and

$$
E_{N}^{\min }\left(\boldsymbol{H}^{\infty}\left(\mathcal{D}_{d}, \omega\right)\right) \geq C_{d, \mu}^{\prime} \exp \left(-\frac{\pi d N}{\log (2 d N / \mu)}\right)
$$

where $C_{d, \mu}^{\prime}$ is another constant depending on $d$ and $\mu$. 
(d) The function

$$
\omega(z)=\exp (-A \cosh (B z)) \quad(A, B>0)
$$

satisfies all the conditions in Theorem 3.2 with $\beta_{1}=(A+\varepsilon) / 2(\varepsilon$ an arbitrarily small positive number), $\beta_{2}=A / 2$, and $\gamma=B$, provided $d<\pi /(2 B)$. Hence Theorem 3.2 yields

$$
E_{N, h}^{\text {sinc }}\left(\boldsymbol{H}^{\infty}\left(\mathcal{D}_{d}, \omega\right)\right) \leq C_{d, A, B} \exp \left(-\frac{\pi d B N}{2 \log (\pi d B N / A)}\right),
$$

where $N=2 n+1, h=\log (2 \pi d B n / A) /(B n)$ and $C_{d, A, B}$ is a constant depending on $d, A$ and $B$, and

$$
E_{N}^{\min }\left(\boldsymbol{H}^{\infty}\left(\mathcal{D}_{d}, \omega\right)\right) \geq C_{d, A, B}^{\prime}(\varepsilon) \exp \left(-\frac{\pi d B N}{\log (\pi d B N /(A+\varepsilon))}\right),
$$

where $C_{d, A, B}^{\prime}(\varepsilon)$ is a constant depending on $d, A, B$ and $\varepsilon$.

Remark 3.2. Let us compare our result with the existing results described in Theorem 1.1. As the special case of Example (a) with $d=\pi / 4$ and $\mu=2$, we have

$$
E_{N, h}^{\mathrm{sinc}}\left(\boldsymbol{H}^{\infty}\left(\mathcal{D}_{\pi / 4}, \omega\right)\right) \leq C \sqrt{N} \exp \left(-\frac{\pi}{2} \sqrt{N}\right),
$$

where $N=2 n+1, h=(\pi / 2) / \sqrt{2 n}$, and

$$
\mathcal{E}_{N}^{\min }\left(\boldsymbol{H}^{\infty}\left(\mathcal{D}_{\pi / 4}, \omega\right)\right) \geq C^{\prime} \exp \left(-\pi \sqrt{\frac{N}{2}}\right) .
$$

The upper estimate above is identical to the upper estimate in Theorem 1.1. On the other hand the lower bound above is an improvement of the lower one in Theorem 1.1. And it is, furthermore, the exact bound, which will be proved later in Section 5 .

Remark 3.3. A careful examination of the proofs, to be given below, of the theorems shows that $\varepsilon$ can be taken to be zero in the estimate for $E_{N}^{\min }$ in (d). That is, in (d) we have

$$
E_{N}^{\min }\left(\boldsymbol{H}^{\infty}\left(\mathcal{D}_{d}, \omega\right)\right) \geq C_{d, A, B}^{\prime} \exp \left(-\frac{\pi d B N}{\log (\pi d B N / A)}\right),
$$

where $C_{d, A, B}^{\prime}$ is a constant depending on $d, A$ and $B$.

\section{Proofs}

4.1. Upper estimate for the sinc approximation formula. We first prove a lemma giving an upper estimate for the error norm of the sinc approximation formula with the mesh size $h$ unspecified. The mesh size is then chosen to optimize the derived upper estimate. We remark that this proof techniques has been employed in [19] in the proof of the upper estimate for the error norm of the trapezoidal formula. 
Lemma 4.1. 1. Suppose that $\omega(z)$ satisfies all the conditions in Theorem [3.1. Then, for any $h>0$,

$$
\begin{aligned}
& E_{N, h}^{\mathrm{sinc}}\left(\boldsymbol{H}^{\infty}\left(\mathcal{D}_{d}, \omega\right)\right) \\
& \quad \leq \frac{\exp (-\pi d / h)}{\pi d(1-\exp (-2 \pi d / h))} \mathcal{N}_{1}\left(\omega, \mathcal{D}_{d}\right)+\frac{2 \alpha_{2} \exp \left(-(\beta h n)^{\rho}\right)}{\rho(\beta h)^{\rho} n^{\rho-1}} .
\end{aligned}
$$

2. Suppose that $\omega(z)$ satisfies all the conditions in Theorem 3.2 Then, for any $h>0$,

$$
\begin{aligned}
& E_{N, h}^{\operatorname{sinc}}\left(\boldsymbol{H}^{\infty}\left(\mathcal{D}_{d}, \omega\right)\right) \\
& \quad \leq \frac{\exp (-\pi d / h)}{\pi d(1-\exp (-2 \pi d / h))} \mathcal{N}_{1}\left(\omega, \mathcal{D}_{d}\right)+\frac{2 \alpha_{2} \exp \left(-\beta_{2} \exp (\gamma h n)\right)}{\beta_{2} \gamma h \exp (\gamma h n)} .
\end{aligned}
$$

The following is crucial to the proof of Lemma 4.1.

Lemma 4.2. Suppose that $\omega(z)$ satisfies conditions 1 and 2 in Theorem 3.1 or Theorem 3.2. Then, for any $h>0$,

$$
E_{N, h}^{\operatorname{sinc}}\left(\boldsymbol{H}^{\infty}\left(\mathcal{D}_{d}, \omega\right)\right) \leq \frac{\exp (-\pi d / h)}{\pi d(1-\exp (-2 \pi d / h))} \mathcal{N}_{1}\left(\omega, \mathcal{D}_{d}\right)+\sum_{|j|>n}|\omega(j h)| .
$$

Proof of Lemma 4.2. The inequality (2.1) and the condition 1 for $\omega(z)$, i.e., $\omega(z) \in$ $B\left(\mathcal{D}_{d}\right)$, imply that any function $f(z) \in \boldsymbol{H}^{\infty}\left(\mathcal{D}_{d}, \omega\right)$ belongs to $B\left(\mathcal{D}_{d}\right)$. For $f(z) \in$ $B\left(\mathcal{D}_{d}\right)$, in turn, the following error estimate of the sinc approximation formula is standard (see Stenger [15, 17]):

$$
\begin{array}{rl}
\sup _{x \in \mathbf{R}} \mid f(x)-\sum_{j=-n}^{n} & f(j h) S(j, h)(x) \mid \\
\leq \sup _{x \in \mathbf{R}}\left|f(x)-\sum_{j=-\infty}^{\infty} f(j h) S(j, h)(x)\right|+\sum_{|j|>n}|f(j h)| \\
\leq \frac{\exp (-\pi d / h)}{\pi d(1-\exp (-2 \pi d / h))} \mathcal{N}_{1}\left(f, \mathcal{D}_{d}\right)+\sum_{|j|>n}|f(j h)| .
\end{array}
$$

Substitution of $|f(j h)| \leq\|f\||\omega(j h)|$ and $\mathcal{N}_{1}\left(f, \mathcal{D}_{d}\right) \leq\|f\| \mathcal{N}_{1}\left(\omega, \mathcal{D}_{d}\right)$, which are easily derived from (2.1), into the right-hand side yields (4.3).

Proof of Lemma 4.1. Under condition 3 for $\omega(z)$ in Theorem 3.1 or in Theorem 3.2 the second term on the right-hand side of (4.3) is estimated as follows.

The case of Theorem 3.1 .

$$
\begin{aligned}
\sum_{|j|>n}|\omega(j h)| & \leq 2 \alpha_{2} \sum_{j=n+1}^{\infty} \exp \left(-(\beta j h)^{\rho}\right) \\
& \leq 2 \alpha_{2} \int_{n}^{\infty} \exp \left(-(\beta h x)^{\rho}\right) d x \\
& \leq \frac{2 \alpha_{2}}{\rho(\beta h)^{\rho} n^{\rho-1}} \int_{n}^{\infty} \rho(\beta h)^{\rho} x^{\rho-1} \exp \left(-(\beta h x)^{\rho}\right) d x \\
& =\frac{2 \alpha_{2} \exp \left(-(\beta h n)^{\rho}\right)}{\rho(\beta h)^{\rho} n^{\rho-1}} .
\end{aligned}
$$


The case of Theorem 3.2 ,

$$
\begin{aligned}
\sum_{|j|>n}|\omega(j h)| & \leq 2 \alpha_{2} \sum_{j=n+1}^{\infty} \exp \left(-\beta_{2} \exp (\gamma j h)\right) \\
& \leq 2 \alpha_{2} \int_{n}^{\infty} \exp \left(-\beta_{2} \exp (\gamma h x)\right) d x \\
& \leq \frac{2 \alpha_{2}}{\beta_{2} \gamma h \exp (\gamma h n)} \int_{n}^{\infty} \beta_{2} \gamma h \exp (\gamma h x) \exp \left(-\beta_{2} \exp (\gamma h x)\right) d x \\
& =\frac{2 \alpha_{2} \exp \left(-\beta_{2} \exp (\gamma h n)\right)}{\beta_{2} \gamma h \exp (\gamma h n)} .
\end{aligned}
$$

Combining these estimates with (4.3), we obtain (4.1) and (4.2) respectively.

Our task is now to choose the mesh size $h$ suitably for a given $n$ and then substitute the chosen mesh size $h$ into the upper estimate for $E_{N, h}^{\text {sinc }}$ which is given in Lemma 4.1

The case of Theorem 3.1. Obviously we should choose the mesh size $h$ to balance the magnitude of the first term with that of the second term on the right-hand side of (4.1). This requirement leads to the equation

$$
\exp (-\pi d / h)=\exp \left(-(\beta h n)^{\rho}\right)
$$

Solving (4.4) for $h$, we have

$$
h=(\pi d)^{\frac{1}{\rho+1}}(\beta n)^{-\frac{\rho}{\rho+1}} .
$$

Then substituting $h$ into the first and the second term on the right-hand side of (4.1), respectively, we obtain

$$
\begin{aligned}
\frac{\exp (-\pi d / h)}{\pi d(1-\exp (-2 \pi d / h))} \mathcal{N}_{1}\left(\omega, \mathcal{D}_{d}\right) & \leq C_{1} \exp \left(-(\pi d \beta n)^{\frac{\rho}{\rho+1}}\right) \\
& \leq C_{1}^{\prime} \exp \left(-\left(\frac{\pi d \beta N}{2}\right)^{\frac{\rho}{\rho+1}}\right) \\
\frac{2 \alpha_{2} \exp \left(-(\beta h n)^{\rho}\right)}{\rho(\beta h)^{\rho} n^{\rho-1}} & \leq C_{2} n^{\frac{\rho}{\rho+1}} \exp \left(-(\pi d \beta n)^{\frac{\rho}{\rho+1}}\right) \\
& \leq C_{2}^{\prime} N^{\frac{\rho}{\rho+1}} \exp \left(-\left(\frac{\pi d \beta N}{2}\right)^{\frac{\rho}{\rho+1}}\right),
\end{aligned}
$$

which yields (3.1), i.e., the upper estimate for $E_{N, h}^{\mathrm{sinc}}$ in Theorem 3.1 .

The case of Theorem 3.2. Similarly we are led to the equation

$$
\exp (-\pi d / h)=\exp \left(-\beta_{2} \exp (\gamma h n)\right) \text {. }
$$

Solving (4.5) for $h$, we have

$$
h=\frac{\log \left(\pi d \gamma n / \beta_{2}\right)}{\gamma n}+\mathrm{O}\left(\frac{\log \log \left(\pi d \gamma n / \beta_{2}\right)}{\gamma n}\right) \quad(n \rightarrow \infty) .
$$

We here choose the mesh size $h$ as

$$
h=\frac{\log \left(\pi d \gamma n / \beta_{2}\right)}{\gamma n}
$$


which is the dominant term in the above asymptotic expansion for $h$. Substituting this $h$ into the first and the second term on the right-hand side of (4.2), respectively, we obtain

$$
\begin{aligned}
\frac{\exp (-\pi d / h)}{\pi d(1-\exp (-2 \pi d / h))} \mathcal{N}_{1}\left(\omega, \mathcal{D}_{d}\right) & \leq C_{3} \exp \left(-\frac{\pi d \gamma n}{\log \left(\pi d \gamma n / \beta_{2}\right)}\right) \\
& \leq C_{3}^{\prime} \exp \left(-\frac{\pi d \gamma N}{2 \log \left(\pi d \gamma N /\left(2 \beta_{2}\right)\right)}\right) \\
\frac{2 \alpha_{2} \exp \left(-\beta_{2} \exp (\gamma h n)\right)}{\beta_{2} \gamma h \exp (\gamma h n)} & =\frac{2 \alpha_{2} \exp (-\pi d \gamma n)}{\pi d \gamma \log \left(\pi d \gamma n / \beta_{2}\right)} \\
& \leq C_{4} \frac{\exp (-\pi d \gamma N / 2)}{\log N}
\end{aligned}
$$

which yields (3.3), i.e., the upper estimate for $E_{N, h}^{\text {sinc }}$ in Theorem 3.2 We have completed the proof of the upper estimates for the error norm of the sinc approximation formula.

4.2. Lower estimate for the minimum error norm. For $z \in \mathbf{C}$ and $\boldsymbol{a}=$ $\left(a_{1}, \ldots, a_{N}\right), a_{j} \in \mathcal{D}_{d}(j=1, \ldots, N)$, define

$$
B_{N}\left(z ; \boldsymbol{a}, \mathcal{D}_{d}\right)=\prod_{i=1}^{N} \frac{T(z)-T\left(a_{i}\right)}{1-\overline{T\left(a_{i}\right)} T(z)},
$$

where

$$
T(z)=\tanh \left(\frac{\pi}{4 d} z\right)
$$

Following [19], we call $B_{N}\left(x ; \boldsymbol{a}, \mathcal{D}_{d}\right)$ the transformed Blaschke product. Note that $T(z)$ is a conformal mapping of the region $\mathcal{D}_{d}$ onto the unit disc.

Using the transformed Blaschke product, we first represent the minimum error norm $E_{N}^{\min }\left(\boldsymbol{H}^{\infty}\left(\mathcal{D}_{d}, \omega\right)\right)$ in a form that is easy to handle.

Lemma 4.3. Suppose that $\omega(z)$ satisfies conditions 1 and 2 in Theorem 3.1 or Theorem 3.2. Then

$$
E_{N}^{\min }\left(\boldsymbol{H}^{\infty}\left(\mathcal{D}_{d}, \omega\right)\right)=\inf _{a_{i} \in \mathbf{R}}\left\{\sup _{x \in \mathbf{R}}\left|B_{N}\left(x ; \boldsymbol{a}, \mathcal{D}_{d}\right) \omega(x)\right|\right\} .
$$

Proof. We establish the inequality

$$
E_{N}^{\min }\left(\boldsymbol{H}^{\infty}\left(\mathcal{D}_{d}, \omega\right)\right) \leq \inf _{a_{i} \in \mathbf{R}}\left\{\sup _{x \in \mathbf{R}}\left|B_{N}\left(x ; \boldsymbol{a}, \mathcal{D}_{d}\right) \omega(x)\right|\right\}
$$

and the converse inequality

$$
E_{N}^{\min }\left(\boldsymbol{H}^{\infty}\left(\mathcal{D}_{d}, \omega\right)\right) \geq \inf _{a_{i} \in \mathbf{R}}\left\{\sup _{x \in \mathbf{R}}\left|B_{N}\left(x ; \boldsymbol{a}, \mathcal{D}_{d}\right) \omega(x)\right|\right\} .
$$


First we establish the inequality (4.7) by proving the following series of inequalities:

$$
\begin{aligned}
E_{N}^{\min } & \left(\boldsymbol{H}^{\infty}\left(\mathcal{D}_{d}, \omega\right)\right) \\
& \leq \inf _{a_{j} \in \mathbf{R}} \inf _{\phi_{j}}\left[\sup _{\| \text {istinct }}\left\{\sup _{x \in \mathbf{R}}\left|f(x)-\sum_{j=1}^{N} f\left(a_{j}\right) \phi_{j}(x)\right|\right\}\right] \\
& \leq \inf _{a_{j} \in \mathbf{R}}\left[\sup _{\| \operatorname{distinct}}\left\{\sup _{x \in \mathbf{R}}\left|f(x)-\sum_{j=1}^{N} f\left(a_{j}\right) \frac{B_{N ; j}\left(x ; \boldsymbol{a}, \mathcal{D}_{d}\right) \omega(x)}{B_{N ; j}\left(a_{j} ; \boldsymbol{a}, \mathcal{D}_{d}\right) \omega\left(a_{j}\right)} T^{\prime}\left(a_{j}-x\right)\right|\right\}\right] \\
& \leq \inf _{a_{j} \in \mathbf{R}}\left\{\sup _{x \in \mathbf{R}}\left|B_{N}\left(x ; \boldsymbol{a}, \mathcal{D}_{d}\right) \omega(x)\right|\right\} \\
& =\operatorname{dinf}_{a_{j} \in \mathbf{R}}\left\{\sup _{x \in \mathbf{R}}\left|B_{N}\left(x ; \boldsymbol{a}, \mathcal{D}_{d}\right) \omega(x)\right|\right\}
\end{aligned}
$$

where, on the right-hand side of the second inequality,

$$
B_{N ; j}\left(z ; \boldsymbol{a}, \mathcal{D}_{d}\right)=\prod_{\substack{i=1 \\ i \neq j}}^{N} \frac{T(z)-T\left(a_{i}\right)}{1-\overline{T\left(a_{i}\right)} T(z)}
$$

The first and the second inequality are trivial. To prove the third inequality, we first observe that for $\delta$ with $0<\delta<d$ and for $a_{j} \in \mathbf{R}(j=1, \ldots, N)$ we have the equality

$$
\begin{aligned}
f(x)-\sum_{j=1}^{N} f\left(a_{j}\right) \frac{B_{N ; j}\left(x ; \boldsymbol{a}, \mathcal{D}_{d}\right) \omega(x)}{B_{N ; j}\left(a_{j} ; \boldsymbol{a}, \mathcal{D}_{d}\right) \omega\left(a_{j}\right)} T^{\prime}\left(a_{j}-x\right) & \\
= & \frac{1}{2 \pi i} \int_{\partial \mathcal{D}_{\delta}} f(\zeta) \frac{B_{N}\left(x ; \boldsymbol{a}, \mathcal{D}_{d}\right) \omega(x)}{B_{N}\left(\zeta ; \boldsymbol{a}, \mathcal{D}_{d}\right) \omega(\zeta)} \frac{T^{\prime}(\zeta-x)}{T(\zeta-x)} d \zeta,
\end{aligned}
$$

which immediately follows from Cauchy's integral formula and condition 1 for $\omega(z)$. Then we have

$$
\begin{aligned}
\sup _{x \in \mathbf{R}} \mid & f(x)-\sum_{j=1}^{N} f\left(a_{j}\right) \frac{B_{N ; j}\left(x ; \boldsymbol{a}, \mathcal{D}_{d}\right) \omega(x)}{B_{N ; j}\left(a_{j} ; \boldsymbol{a}, \mathcal{D}_{d}\right) \omega\left(a_{j}\right)} T^{\prime}\left(a_{j}-x\right) \mid \\
\leq & \varlimsup_{\delta \uparrow d}\left\{\sup _{x \in \mathbf{R}}\left|\frac{1}{2 \pi i} \int_{\partial \mathcal{D}_{\delta}} \frac{f(\zeta)}{\omega(\zeta)} \frac{B_{N}\left(x ; \boldsymbol{a}, \mathcal{D}_{d}\right) \omega(x)}{B_{N}\left(\zeta ; \boldsymbol{a}, \mathcal{D}_{d}\right)} \frac{T^{\prime}(\zeta-x)}{T(\zeta-x)} d \zeta\right|\right\} \\
\leq & \|f\| \sup _{x \in \mathbf{R}}\left|B_{N}\left(x ; \boldsymbol{a}, \mathcal{D}_{d}\right) \omega(x)\right| \\
& \quad \times \varlimsup_{\delta \uparrow d}\left[\frac{1}{\inf _{\zeta \in \partial \mathcal{D}_{\delta}}\left|B_{N}\left(\zeta ; \boldsymbol{a}, \mathcal{D}_{d}\right)\right|} \sup _{x \in \mathbf{R}}\left\{\frac{1}{2 \pi} \int_{\partial \mathcal{D}_{\delta}} \frac{\left|T^{\prime}(\zeta-x)\right|}{|T(\zeta-x)|}|d \zeta|\right\} \sup _{x \in \mathbf{R}}\left|B_{N}\left(x ; \boldsymbol{a}, \mathcal{D}_{d}\right) \omega(x)\right| \varlimsup_{\delta \uparrow d} \frac{1}{2 \pi} \int_{\partial \mathcal{D}_{\delta}} \frac{\left|T^{\prime}(\zeta)\right|}{|T(\zeta)|}|d \zeta|\right. \\
= & \|f\| \sup _{x \in \mathbf{R}}\left|B_{N}\left(x ; \boldsymbol{a}, \mathcal{D}_{d}\right) \omega(x)\right| \varlimsup_{\delta \uparrow d} \frac{1}{2 \pi} \int_{T\left(\partial \mathcal{D}_{\delta}\right)} \frac{1}{|z|}|d z| \\
= & \|f\| \sup _{x \in \mathbf{R}}\left|B_{N}\left(x ; \boldsymbol{a}, \mathcal{D}_{d}\right) \omega(x)\right|,
\end{aligned}
$$


which implies the third inequality. The fourth equality immediately follows from the fact that $B_{N}\left(x ; \boldsymbol{a}, \mathcal{D}_{d}\right)$ is a continuous function of $\boldsymbol{a}$. Thus we have established the inequality (4.7).

Next we establish the inequality (4.8) by proving the following series of inequalities:

$$
\begin{aligned}
E_{N}^{\min } & \left(\boldsymbol{H}^{\infty}\left(\mathcal{D}_{d}, \omega\right)\right) \\
& \geq \inf _{1 \leq l \leq N} \inf _{\substack{m_{1}, m_{2}, \ldots, m_{l} \\
m_{1}+m_{2}+\cdots+m_{l}=N}} \inf _{\substack{a_{j} \in \mathcal{D}_{d} \\
\text { distinct }}} \inf _{\phi_{j k}}\left[\sup _{f \in \boldsymbol{F}_{0}\left(\left\{a_{j}\right\},\left\{m_{j}\right\}\right)}\left\{\sup _{x \in \mathbf{R}}|f(x)|\right\}\right] \\
& \geq \inf _{a_{i} \in \mathcal{D}_{d}}\left\{\sup _{x \in \mathbf{R}}\left|B_{N}\left(x ; \boldsymbol{a}, \mathcal{D}_{d}\right) \omega(x)\right|\right\} \\
& =\inf _{a_{i} \in \mathbf{R}}\left\{\sup _{x \in \mathbf{R}}\left|B_{N}\left(x ; \boldsymbol{a}, \mathcal{D}_{d}\right) \omega(x)\right|\right\},
\end{aligned}
$$

where, on the right-hand side of the first inequality, $\boldsymbol{F}_{0}\left(\left\{a_{j}\right\},\left\{m_{j}\right\}\right)$ is the subspace of $\boldsymbol{H}^{\infty}\left(\mathcal{D}_{d}, \omega\right)$ defined by

$$
\begin{aligned}
& \boldsymbol{F}_{0}\left(\left\{a_{j}\right\},\left\{m_{j}\right\}\right) \\
& \quad=\left\{f \in \boldsymbol{H}^{\infty}\left(\mathcal{D}_{d}, \omega\right) \mid\|f\| \leq 1 \text { and } f^{(k)}\left(a_{j}\right)=0, k=0, \ldots, m_{j}-1, j=1, \ldots, l\right\} .
\end{aligned}
$$

The first inequality is trivial. The second one is evident from the fact that, with $\boldsymbol{a}=\left(a_{1}, \ldots, a_{1}, \ldots, a_{l}, \ldots, a_{l}\right)$, where each $a_{j}$ is repeated $m_{j}$ times, the transformed Blaschke product $B_{N}\left(z ; \boldsymbol{a}, \mathcal{D}_{d}\right)$ belongs to $\boldsymbol{F}_{0}\left(\left\{a_{j}\right\},\left\{m_{j}\right\}\right)$. The third equality is also evident from the inequality

$$
\left|\frac{\xi-\alpha}{1-\bar{\alpha} \xi}\right| \geq\left|\frac{\xi-\operatorname{Re} \alpha}{1-(\operatorname{Re} \alpha) \xi}\right| \quad(-1<\xi<1,|\alpha|<1),
$$

which is easily verified by a direct calculation.

Owing to Lemma 4.3 the following key lemma is easily obtained.

Lemma 4.4. Suppose that $\omega(z)$ satisfies conditions 1 and 2 in Theorem 3.1 or [3.2. Then

$$
E_{N}^{\min }\left(\boldsymbol{H}^{\infty}\left(\mathcal{D}_{d}, \omega\right)\right) \geq \sup _{R \in \mathbf{R}} \exp \left(-\frac{\pi d N}{2 R}+\frac{1}{2 R} \int_{-R}^{R} \log |\omega(x)| d x\right) .
$$

Proof. The right-hand side of (4.6) is bounded from below as follows:

$$
\begin{aligned}
\inf _{a_{i} \in \mathbf{R}} & \left\{\sup _{x \in \mathbf{R}}\left|B_{N}\left(x ; \boldsymbol{a}, \mathcal{D}_{d}\right) \omega(x)\right|\right\} \\
& \geq \inf _{a_{i} \in \mathbf{R}}\left\{\sup _{R \in \mathbf{R}} \int_{-R}^{R}\left|B_{N}\left(x ; \boldsymbol{a}, \mathcal{D}_{d}\right) \omega(x)\right| \frac{d x}{2 R}\right\} .
\end{aligned}
$$

Based on the same techniques as used in [2, 19], namely, Jensen's inequality and Newman's inequality 12

$$
\int_{-\rho}^{\rho} \log \left|\frac{\xi-\alpha}{1-\bar{\alpha} \xi}\right| \frac{d \xi}{1-\xi^{2}} \geq-\frac{\pi^{2}}{4} \quad(0 \leq \rho \leq 1,|\alpha|<1),
$$


we estimate the integral on the right-hand side as follows:

$$
\begin{aligned}
\int_{-R}^{R} \mid & B_{N}\left(x ; \boldsymbol{a}, \mathcal{D}_{d}\right) \omega(x) \mid \frac{d x}{2 R} \\
\quad & \geq \exp \left\{\int_{-R}^{R} \log \left(\left|B_{N}\left(x ; \boldsymbol{a}, \mathcal{D}_{d}\right)\right||\omega(x)|\right) \frac{d x}{2 R}\right\} \\
\quad & \exp \left[\frac{d}{\pi R} \int_{-T(R)}^{T(R)} \log \prod_{i=1}^{N}\left|\frac{\xi-T\left(a_{i}\right)}{1-\overline{T\left(a_{i}\right)} \xi}\right| \frac{d \xi}{1-\xi^{2}}+\frac{1}{2 R} \int_{-R}^{R} \log |\omega(x)| d x\right] \\
& \geq \exp \left(-\frac{\pi d N}{2 R}+\frac{1}{2 R} \int_{-R}^{R} \log |\omega(x)| d x\right) .
\end{aligned}
$$

The substitution of the resulting inequality into the right-hand side of (4.10) yields the demanded estimate for $E_{N}^{\min }\left(\boldsymbol{H}^{\infty}\left(\mathcal{D}_{d}, \omega\right)\right)$.

The proof of the lower estimates, (3.2) and (3.4), for the minimum error norm is completed by estimating the right-hand side of (4.9).

Lemma 4.5. 1. Suppose that $\omega(z)$ satisfies all the conditions in Theorem 3.1. Then

$$
\begin{aligned}
\sup _{R \in \mathbf{R}} \exp & \left(-\frac{\pi d N}{2 R}+\frac{1}{2 R} \int_{-R}^{R} \log |\omega(x)| d x\right) \\
& \geq \sup _{R \in \mathbf{R}} \exp \left(-\frac{\pi d N}{2 R}+\log \alpha_{1}-\frac{(\beta R)^{\rho}}{\rho+1}\right) \\
& \geq C^{\prime} \exp \left(-\left(\left(\frac{2}{\rho+1}\right)^{1 / \rho} \pi d \beta N\right)^{\rho /(\rho+1)}\right) .
\end{aligned}
$$

2. Suppose that $\omega(z)$ satisfies all the conditions in Theorem 3.2. Then

$$
\begin{aligned}
\sup _{R \in \mathbf{R}} \exp & \left(-\frac{\pi d N}{2 R}+\frac{1}{2 R} \int_{-R}^{R} \log |\omega(x)| d x\right) \\
& \geq \sup _{R \in \mathbf{R}} \exp \left(-\frac{\pi d N}{2 R}+\log \alpha_{1}-\frac{\beta_{1}(\exp (\gamma R)-1)}{\gamma R}\right) \\
& \geq C^{\prime} \exp \left(-\frac{\pi d \gamma N}{\log \left(\pi d \gamma N /\left(2 \beta_{1}\right)\right)}\right) .
\end{aligned}
$$

Proof. In both 1 and 2 the first inequalities are trivial, and the second ones are obtained by taking

$$
R=\left(\frac{(\rho+1) \pi d N}{2 \beta^{\rho}}\right)^{1 /(\rho+1)}
$$

and

$$
R=\frac{1}{\gamma} \log \left(\frac{\pi d \gamma N}{2 \beta_{1}}\right)
$$

respectively. 


\section{FinAL REMARKS}

Some remarks are added on subjects or problems closely related to the present work.

1. There remains a theoretical problem of determining the exact order of the minimum error norm of the approximation in the spaces $\boldsymbol{H}^{\infty}\left(\mathcal{D}_{d}, \omega\right)$. It should be noted here that the exact order is easily obtained for the space $\boldsymbol{H}^{\infty}\left(\mathcal{D}_{\pi / 4}, \operatorname{sech}^{\mu}(z)\right)$, which reads as follows:

$$
E_{N}^{\min }\left(\boldsymbol{H}^{\infty}\left(\mathcal{D}_{\pi / 4}, \operatorname{sech}^{\mu}(z)\right)\right) \asymp \exp \left(-\frac{\pi}{2} \sqrt{\mu N}\right) .
$$

We shall give the proof below. It suffice to prove the estimate

$$
C_{\mu} \exp \left(-\frac{\pi}{2} \sqrt{\mu N}\right) \leq E_{N}^{\min }\left(\boldsymbol{H}^{\infty}\left(\mathcal{D}_{\pi / 4}, \operatorname{sech}^{\mu}(z)\right)\right) \leq C_{\mu}^{\prime} \exp \left(-\frac{\pi}{2} \sqrt{\mu N}\right),
$$

where $C_{\mu}$ and $C_{\mu}^{\prime}$ are positive constants depending on $\mu$.

The lower estimate has already been established in Example (a) in Section 2.

For the upper estimate we need the following result of Ganelius ([2, 6]):

There exist $\alpha_{1}, \alpha_{2}, \ldots, \alpha_{N} \in(-1,1)$ such that

$$
\max _{x \in[-1,1]}\left(1-x^{2}\right)^{\mu / 2} \prod_{k=1}^{N}\left|\frac{x-\alpha_{k}}{1+\alpha_{k} x}\right| \leq C_{\mu}^{\prime} \exp \left(-\frac{\pi}{2} \sqrt{\mu N}\right),
$$

where $C_{\mu}^{\prime}$ is a positive constant depending on $\mu$.

Transforming this result to the strip region $\mathcal{D}_{\pi / 4}$, we can find the transformed Blaschke product $B_{N}\left(x ; \boldsymbol{a}, \mathcal{D}_{\pi / 4}\right)$ such that

$$
\sup _{x \in \mathbf{R}}\left|B_{N}\left(x ; \boldsymbol{a}, \mathcal{D}_{\pi / 4}\right)\right| \operatorname{sech}^{\mu}(x) \leq C_{\mu}^{\prime} \exp \left(-\frac{\pi}{2} \sqrt{\mu N}\right) .
$$

So it follows that

$$
\inf _{a_{i} \in \mathbf{R}}\left\{\sup _{x \in \mathbf{R}}\left|B_{N}\left(x ; \boldsymbol{a}, \mathcal{D}_{\pi / 4}\right)\right| \operatorname{sech}^{\mu}(x)\right\} \leq C_{\mu}^{\prime} \exp \left(-\frac{\pi}{2} \sqrt{\mu N}\right),
$$

which yields the demanded upper estimate.

2. Up to the present there have been proposed a variety of sinc approximation formulas obtained by variable transformation. Typical examples are the following:

(1) Sinc approximation formulas obtained by ordinary transformations 1

$$
\begin{aligned}
& f(x) \approx \sum_{j=-n}^{n} f(\tanh (j h)) S(j, h)\left(\tanh ^{-1}(x)\right), \\
& f(x) \approx \sum_{j=-n}^{n} f(\exp (j h)) S(j, h)(\log (x)), \\
& f(x) \approx \sum_{j=-n}^{n} f\left(\sinh ^{-1}(\exp (j h))\right) S(j, h)(\log (\sinh (x))), \\
& f(x) \approx \sum_{j=-n}^{n} f(\sinh (j h)) S(j, h)\left(\sinh ^{-1}(x)\right) ;
\end{aligned}
$$

\footnotetext{
${ }^{1}$ The variable transformations used in the formulas (5.6, (5.7), and (5.8) are collectively called double exponential transformations, but the variable transformations used in (5.2), (5.3), (5.4) and [5.5) have no definite names. Following Stenger [18], we here call them "ordinary transformations" collectively.
} 
(2) Sinc approximation formulas obtained by double exponential transformations: 2

$$
\begin{aligned}
& f(x) \approx \sum_{j=-n}^{n} f\left(\tanh \left(\frac{\pi}{2} \sinh (j h)\right)\right) S(j, h)\left(\sinh ^{-1}\left(\frac{2}{\pi} \tanh ^{-1}(x)\right)\right), \\
& f(x) \approx \sum_{j=-n}^{n} f\left(\exp \left(\frac{\pi}{2} \sinh (j h)\right)\right) S(j, h)\left(\sinh ^{-1}\left(\frac{2}{\pi} \log (x)\right)\right), \\
& f(x) \approx \sum_{j=-n}^{n} f\left(\sinh \left(\frac{\pi}{2} \sinh (j h)\right)\right) S(j, h)\left(\sinh ^{-1}\left(\frac{2}{\pi} \sinh ^{-1}(x)\right)\right) .
\end{aligned}
$$

It should be noted here that their near-optimality in suitable spaces is established by employing the results of Section 3. In fact, the general procedure is given as below, for establishing the near-optimality of the sinc approximation formulas obtained by the variable transformation $\tau$ :

$$
f(y) \approx \sum_{j=-n}^{n} f(\tau(j h)) S(j, h)\left(\tau^{-1}(y)\right) \quad(y \in \tau(\mathbf{R})) .
$$

Step 1. Define the function space $\boldsymbol{H}_{\tau}^{\infty *}\left(\mathcal{D}_{d}, \omega\right)$ by

$$
\boldsymbol{H}_{\tau}^{\infty *}\left(\mathcal{D}_{d}, \omega\right)=\left\{f(w): \tau\left(\mathcal{D}_{d}\right) \rightarrow \mathbf{C} \mid f(\tau(z)) \in \boldsymbol{H}^{\infty}\left(\mathcal{D}_{d}, \omega\right)\right\},
$$

and define the norm \|\|$_{\tau}$ on $\boldsymbol{H}_{\tau}^{\infty *}\left(\mathcal{D}_{d}, \omega\right)$ by

$$
\|f\|_{\tau} \equiv\|(f \circ \tau)\|
$$

where \|\| on the right-hand side is the norm on $\boldsymbol{H}^{\infty}\left(\mathcal{D}_{d}, \omega\right)$.

For the sinc approximation formula obtained by the variable transformation $\tau$, let $E_{N, h}^{\text {sinc, } \tau}\left(\boldsymbol{H}_{\tau}^{\infty *}\left(\mathcal{D}_{d}, \omega\right)\right)$ denote the error norm in $\boldsymbol{H}_{\tau}^{\infty *}\left(\mathcal{D}_{d}, \omega\right)$ :

$$
E_{N, h}^{\text {sinc }, \tau}\left(\boldsymbol{H}_{\tau}^{\infty *}\left(\mathcal{D}_{d}, \omega\right)\right)=\sup _{\|f\|_{\tau} \leq 1}\left\{\sup _{y \in \tau(\mathbf{R})}\left|f(y)-\sum_{j=-n}^{n} f(\tau(j h)) S(j, h)\left(\tau^{-1}(y)\right)\right|\right\}
$$

and let $E_{N}^{\min }\left(\boldsymbol{H}_{\tau}^{\infty *}\left(\mathcal{D}_{d}, \omega\right)\right)$ designate the minimum error norm in $\boldsymbol{H}_{\tau}^{\infty *}\left(\mathcal{D}_{d}, \omega\right)$, where the minimum is taken over all the $N$-point approximation formulas:

$$
\begin{aligned}
& E_{N}^{\min }\left(\boldsymbol{H}_{\tau}^{\infty *}\left(\mathcal{D}_{d}, \omega\right)\right) \\
& =\inf _{1 \leq l \leq N} \inf _{\substack{m_{1}, m_{2}, \ldots, m_{l} \\
m_{1}+m_{2}+\cdots+m_{l}=N}} \inf _{b_{j} \in \tau\left(\mathcal{D}_{d}\right)} \inf _{\substack{\text { distinct } \\
\psi_{j k}}}\left\{\sup _{\|f\|_{\tau} \leq 1}\left\{\sup _{y \in \tau(\mathbf{R})}\left|f(y)-\sum_{j=1}^{l} \sum_{k=0}^{m_{j}-1} f^{(k)}\left(b_{j}\right) \psi_{j k}(y)\right|\right\}\right\} .
\end{aligned}
$$

Step 2. Claim the near-optimality of the sinc approximation formula obtained by the variable transformation $\tau$ in the space $\boldsymbol{H}_{\tau}^{\infty *}\left(\mathcal{D}_{d}, \omega\right)$, i.e.,

$$
E_{N, h}^{\operatorname{sinc}, \tau}\left(\boldsymbol{H}_{\tau}^{\infty *}\left(\mathcal{D}_{d}, \omega\right)\right) \approx E_{N}^{\min }\left(\boldsymbol{H}_{\tau}^{\infty *}\left(\mathcal{D}_{d}, \omega\right)\right)
$$

\footnotetext{
${ }^{2}$ The double exponential transformations were first proposed by Takahasi and Mori in 1974 for numerical integration 20. Recently it turned out that the double exponential transformations are useful not only for numerical integration but also for various kinds of sinc numerical methods (see Mori and Sugihara 11).
} 
based on the results of Section 3 and the following key observations:

- The error norm of the sinc approximation formula obtained by the variable transformation $\tau$ in the space $\boldsymbol{H}_{\tau}^{\infty *}\left(\mathcal{D}_{d}, \omega\right)$ is identical to the error norm of the sinc approximation formula in the space $\boldsymbol{H}^{\infty}\left(\mathcal{D}_{d}, \omega\right)$ :

$$
E_{N, h}^{\mathrm{sinc}, \tau}\left(\boldsymbol{H}_{\tau}^{\infty *}\left(\mathcal{D}_{d}, \omega\right)\right)=E_{N, h}^{\mathrm{sinc}}\left(\boldsymbol{H}^{\infty}\left(\mathcal{D}_{d}, \omega\right)\right) .
$$

- The minimum error norm of the $N$-point approximation formula in the space $\boldsymbol{H}_{\tau}^{\infty *}\left(\mathcal{D}_{d}, \omega\right)$ is identical to the minimum error norm of the $N$-point approximation formula in the space $\boldsymbol{H}^{\infty}\left(\mathcal{D}_{d}, \omega\right)$ :

$$
E_{N}^{\min }\left(\boldsymbol{H}_{\tau}^{\infty *}\left(\mathcal{D}_{d}, \omega\right)\right)=E_{N}^{\min }\left(\boldsymbol{H}^{\infty}\left(\mathcal{D}_{d}, \omega\right)\right) .
$$

The procedure above yields a variety of near-optimality results for the sinc approximation obtained by variable transformation. In what follows, we shall describe some of these.

(a) Set $\omega(z)=\operatorname{sech}^{\mu}(z)$ and $\tau(z)=\tanh (z)$ in the procedure above. Then, for (5.2) we have

$$
\begin{aligned}
C_{d, \mu} \sqrt{N} \exp \left(-\sqrt{\frac{\pi d \mu N}{2}}\right) & \geq E_{N, h}^{\operatorname{sinc}, \tau}\left(\boldsymbol{H}_{\tau}^{\infty *}\left(\mathcal{D}_{d}, \omega\right)\right) \\
& \geq E_{N}^{\min }\left(\boldsymbol{H}_{\tau}^{\infty *}\left(\mathcal{D}_{d}, \omega\right)\right) \geq C_{d, \mu}^{\prime} \exp (-\sqrt{\pi d \mu N}) .
\end{aligned}
$$

The space $\boldsymbol{H}_{\tau}^{\infty *}\left(\mathcal{D}_{d}, \omega\right)$ is identical to the space $\boldsymbol{H}_{\infty}^{*}\left(\mathcal{D}_{d}^{\mathrm{E}},\left(1-w^{2}\right)^{\mu / 2}\right)$, where $\boldsymbol{H}_{\infty}^{*}\left(\mathcal{D}_{d}^{\mathrm{E}},\left(1-w^{2}\right)^{\mu / 2}\right)$ is the family of all functions $f$ analytic in the eye-shaped region $\mathcal{D}_{d}^{\mathrm{E}}=\{w \in \mathbf{C} \mid \arg [(1-w) /(1+w)]<d / 2\}$ such that

$$
\|f\|_{\infty} \equiv \sup _{w \in \mathcal{D}_{d}^{E}}\left|f(w) /\left(1-w^{2}\right)^{\mu / 2}\right|<\infty .
$$

This space is a generalization of the $H_{\infty}$ space that was intensively studied by Stenger 14, 16.

(b) Set $\omega(z)=\operatorname{sech}^{\mu}(z)$ and $\tau(z)=\exp (z)$ in the procedure above. Then, for (5.3) we have

$$
\begin{aligned}
C_{d, \mu} \sqrt{N} \exp \left(-\sqrt{\frac{\pi d \mu N}{2}}\right) & \geq E_{N, h}^{\mathrm{sinc}, \tau}\left(\boldsymbol{H}_{\tau}^{\infty *}\left(\mathcal{D}_{d}, \omega\right)\right) \\
& \geq E_{N}^{\min }\left(\boldsymbol{H}_{\tau}^{\infty *}\left(\mathcal{D}_{d}, \omega\right)\right) \geq C_{d, \mu}^{\prime} \exp (-\sqrt{\pi d \mu N}) .
\end{aligned}
$$

The space $\boldsymbol{H}_{\tau}^{\infty *}\left(\mathcal{D}_{d}, \omega\right)$ is identical to the space $\boldsymbol{H}_{\infty}^{*}\left(\mathcal{D}_{d}^{\mathrm{W}},\left(2 w /\left(1+w^{2}\right)\right)^{\mu}\right)$, where $\boldsymbol{H}_{\infty}^{*}\left(\mathcal{D}_{d}^{\mathrm{W}},\left(2 w /\left(1+w^{2}\right)\right)^{\mu}\right)$ is the family of all functions $f$ analytic in the region $\mathcal{D}_{d}^{\mathrm{W}}$ $=\{w \in \mathbf{C}|| \arg w \mid<d\}$ such that

$$
\|f\|_{\infty} \equiv \sup _{w \in \mathcal{D}_{d}^{\mathrm{W}}}\left|f(w)\left(\left(1+w^{2}\right) / 2 w\right)^{\mu}\right|<\infty .
$$

(c) Set $\omega(z)=\operatorname{sech}^{\mu}(z)$ and $\tau(z)=\sinh (z)$ in the procedure above. Then, for (5.5) we have

$$
\begin{aligned}
C_{d, \mu} \sqrt{N} \exp \left(-\sqrt{\frac{\pi d \mu N}{2}}\right) & \geq E_{N, h}^{\mathrm{sinc}, \tau}\left(\boldsymbol{H}_{\tau}^{\infty *}\left(\mathcal{D}_{d}, \omega\right)\right) \\
& \geq E_{N}^{\min }\left(\boldsymbol{H}_{\tau}^{\infty *}\left(\mathcal{D}_{d}, \omega\right)\right) \geq C_{d, \mu}^{\prime} \exp (-\sqrt{\pi d \mu N}) .
\end{aligned}
$$


The space $\boldsymbol{H}_{\tau}^{\infty *}\left(\mathcal{D}_{d}, \omega\right)$ is identical to the space $\boldsymbol{H}_{\infty}^{*}\left(\mathcal{D}_{d}^{\mathrm{H}}, 1 /\left(1+w^{2}\right)^{\mu / 2}\right)$, where $\boldsymbol{H}_{\infty}^{*}\left(\mathcal{D}_{d}^{\mathrm{H}}, 1 /\left(1+w^{2}\right)^{\mu / 2}\right)$ is the family of all functions $f$ analytic in the region $\mathcal{D}_{d}^{\mathrm{H}}=\left\{w \in \mathbf{C} \mid w=u+i v, v^{2} / \sin ^{2} d-u^{2} / \cos ^{2} d<1\right\}$ such that

$$
\|f\|_{\infty} \equiv \sup _{w \in \mathcal{D}_{d}^{\mathrm{H}}}\left|f(w)\left(1+w^{2}\right)^{\mu / 2}\right|<\infty .
$$

(d) Set

$$
\omega(z)=\operatorname{sech}^{\mu}\left(\frac{\pi}{2} \sinh (z)\right) \quad \text { and } \quad \tau(z)=\tanh \left(\frac{\pi}{2} \sinh (z)\right)
$$

in the procedure above. Then, for (5.6) we have

$$
\begin{aligned}
C_{d, \mu} \exp \left(-\frac{\pi d N}{2 \log (2 d N / \mu)}\right) & \geq E_{N, h}^{\operatorname{sinc}, \tau}\left(\boldsymbol{H}_{\tau}^{\infty *}\left(\mathcal{D}_{d}, \omega\right)\right) \\
& \geq E_{N}^{\min }\left(\boldsymbol{H}_{\tau}^{\infty *}\left(\mathcal{D}_{d}, \omega\right)\right) \geq C_{d, \mu}^{\prime} \exp \left(-\frac{\pi d N}{\log (2 d N / \mu)}\right) .
\end{aligned}
$$

The space $\boldsymbol{H}_{\tau}^{\infty *}\left(\mathcal{D}_{d}, \omega\right)$ is identical to the space $\boldsymbol{H}_{\infty}^{*}\left(\tau\left(\mathcal{D}_{d}\right),\left(1-w^{2}\right)^{\mu / 2}\right)$, where $\boldsymbol{H}_{\infty}^{*}\left(\tau\left(\mathcal{D}_{d}\right),\left(1-w^{2}\right)^{\mu / 2}\right)$ is the family of all functions $f$ analytic in the infinitely sheeted Riemann surface $\tau\left(\mathcal{D}_{d}\right)=\left\{w=\tau(z) \mid z \in \mathcal{D}_{d}\right\}$ such that

$$
\|f\|_{\infty} \equiv \sup _{w \in \tau\left(\mathcal{D}_{d}\right)}\left|f(w) /\left(1-w^{2}\right)^{\mu / 2}\right|<\infty .
$$

3. The problem of approximating $|x|^{\mu}$ on the interval $[-1,1]$ has been extensively studied in approximation theory. Various methods have been considered, such as polynomials, spline functions (piecewise polynomials), rational functions, as well as various types of sinc functions, and it is known that the best convergence rate is $O(\exp (-\pi n / \log n))$ (where $n$ is the dimension of the space of approximating functions), which is attainted by a sinc approximation formula obtained by variable transformation. We here show that this rate can be improved based on the result obtained in Example (c) in Section 3.

Before proceeding to our result, we briefly mention known results on the approximation to the function $|x|^{\mu}$ by polynomials, by spline functions, and by sinc functions.

Polynomial approximation. The error of the best approximation of $|x|^{\mu}$ on $[-1,1]$ by polynomials was determined by Bernstein [3] to be

$$
E_{n}\left(|x|^{\mu}\right)=O\left(n^{-\mu}\right) \quad \text { as } n \rightarrow \infty,
$$

where $E_{n}(f)$ denotes the error of the best approximation of $f$ on $[-1,1]$ by polynomials of degree $n-1$.

Spline approximation. Rice 13] showed that the error of the best approximation of $|x|^{\mu}$ on $[-1,1]$ by $n$ spline functions of degree $k$ converges to zero at the rate $O\left(n^{-k}\right)$.

For variable knots and variable degree splines, DeVore and Scherer [5] proved that the error of the best approximation by $n$ spline functions (h-p) converges to zero at the rate

$$
O(\exp (-c \sqrt{\mu n})), \quad c=-2 \log (\sqrt{2}-1) \approx 1.7627472 .
$$


Sinc approximation. Gustafson and Stenger [7] showed that the error of the sinc approximation formula using an ordinary-type transformation $x=\tau_{\text {od }}(y)=$ $\operatorname{sign} y / \cosh y$ is estimated by

$$
\begin{aligned}
\left.\sup _{x \in[-1,1]}|| x\right|^{\mu}- & \left|\tau_{\text {od }}(0)\right|^{\mu} S(0, h) \circ \tau_{\text {od }}^{-1}(x) \\
& +\sum_{j=1}^{n}\left|\tau_{\text {od }}(j h)\right|^{\mu}\left\{S(j, h) \circ \tau_{\text {od }}^{-1}(x)+S(-j, h) \circ \tau_{\text {od }}^{-1}(x)\right\} \mid \\
& =O\left(n^{1 / 2} \exp \left(-c^{\prime} \sqrt{\mu n}\right)\right),
\end{aligned}
$$

where $c^{\prime}=\pi / \sqrt{2} \approx 2.2214415$, and $h$ is taken by $h=\pi / \sqrt{2 \mu n}$.

Further, employing a double exponential type transformation $x=\tau_{\text {de }}(y)=$ $\operatorname{sign} y / \cosh (\sinh y)$, Keinert [8] established that

$$
\begin{aligned}
\left.\sup _{x \in[-1,1]}|| x\right|^{\mu}-\left|\tau_{\mathrm{de}}(0)\right|^{\mu} S(0, h) \circ \tau_{\mathrm{de}}^{-1}(x) & \\
+\sum_{j=1}^{n}\left|\tau_{\mathrm{de}}(j h)\right|^{\mu}\left\{S(j, h) \circ \tau_{\mathrm{de}}^{-1}(x)+S(-j, h) \circ \tau_{\mathrm{de}}^{-1}(x)\right\} \mid & =O(\exp (-\pi n / \log n))
\end{aligned}
$$

where $h=\log n / n$, which result is known to be the best one.

Let us now proceed to our result. From the upper estimate for $E_{N, h}^{\mathrm{sinc}}$ in Example (c) in Section 3, we know that, for an arbitrarily small $\varepsilon>0$,

$$
\begin{array}{r}
\sup _{y \in \mathbf{R}}\left|\operatorname{sech}^{\mu}\left(\frac{\pi}{2} \sinh (y)\right)-\sum_{j=-n}^{n} \operatorname{sech}^{\mu}\left(\frac{\pi}{2} \sinh (j h)\right) S(j, h)(y)\right| \\
\leq C_{(\pi-\varepsilon) / 2, \mu} \exp \left(-\frac{\pi(\pi-\varepsilon) N}{4 \log ((\pi-\varepsilon) N / \mu)}\right)
\end{array}
$$

where $N=2 n+1$ and $h=\log (2(\pi-\varepsilon) n / \mu) / n$, and where $C_{(\pi-\varepsilon) / 2, \mu}$ denotes the constant $C_{d, \mu}$ in (3.9) for $d=(\pi-\varepsilon) / 2$. Transforming this result to the interval $[-1,1]$ by another double exponential type transformation $x=\widetilde{\tau}_{\text {de }}(y)=$ $\operatorname{sign} y / \cosh \left(\frac{\pi}{2} \sinh y\right)$, we obtain

$$
\begin{aligned}
& \left.\sup _{x \in[-1,1]}|| x\right|^{\mu}-\left|\widetilde{\tau}_{\mathrm{de}}(0)\right|^{\mu} S(0, h) \circ \widetilde{\tau}_{\mathrm{de}}^{-1}(x) \\
& +\sum_{j=1}^{n}\left|\widetilde{\tau}_{\mathrm{de}}(j h)\right|^{\mu}\left\{S(j, h) \circ \widetilde{\tau}_{\mathrm{de}}^{-1}(x)+S(-j, h) \circ \widetilde{\tau}_{\mathrm{de}}^{-1}(x)\right\} \mid \\
& \quad=O\left(\exp \left(-\frac{\pi(\pi-\varepsilon) n}{2 \log (2(\pi-\varepsilon) n / \mu)}\right)\right) .
\end{aligned}
$$

This result is obviously better than Keinert's. Furthermore, in view of Theorem 3.3 we conjecture that it is almost best, as far as the sinc approximation is concerned. 
4. As is seen in the preceding part, it is obvious that in cases when the double exponential transformation applies, one is able to achieve more rapid convergence of approximation than by the sinc approximation employing the ordinary transformation. It is also important, but often overlooked, that the sinc approximation employing the ordinary transformation applies when the conditions for the double exponential transformation apply, but not necessarily vice versa.

\section{Example.}

$$
f(x)=\left(1-x^{2}\right)^{\mu} \operatorname{cn}\left(\tanh ^{-1} x, 0.5\right), \quad-1 \leq x \leq 1,
$$

where $\mu>0$ and $\operatorname{cn}(x, 0.5)$ is the Jacobi elliptic function which has singularities at $K(2 m+(2 n+1)$ i) where $K=1.85407 \ldots$ and $m$ and $n$ are integers [1]. For the function $f(x)$ the condition for the ordinary transformation $x=\tanh y$ applies, but the sinc approximation employing the double exponential transformation $x=$ $\tanh (\pi / 2(\sinh y))$ does not apply.

\section{ACKNOWLEDGMENTS}

The author wishes to express his deep appreciation to Professor Masatake Mori of Kyoto University for his constant encouragement. The author also wishes to express his gratitude to Professor Kazuo Murota of Kyoto University for his valuable suggestions. The author is grateful to the referee for valuable comments.

\section{REFERENCES}

[1] M. Abramowitz and I. Stegun, Handbook of mathematical functions, NBS Applied Math. Series 53, 1964. MR 29:4914

[2] J.-E. Andersson, Optimal quadrature of $H^{p}$ functions, Math. Z. 172 (1980), 55-62. MR 83m:41023

[3] S. N. Bernstein, Sur la meilleure approximation de $|x|^{p}$ par des polynômes de degrées très élevés, Bull. Acad. Sci. USSR, Cl. Sci. Math. Nat. 2 (1938), 181-190.

[4] H. G. Burchard and K. Höllig, $n$-width and entropy of $H^{p}$-classes in $L_{q}(-1,1)$, SIAM J. Math. Anal. 16 (1985), 405-421. MR 86i:41021

[5] R. A. DeVore and K. Scherer, Variable knot, variable degree spline approximation to $x^{\mu}$, in: Quantitative Approximation (R. A. DeVore and K. Scherer, eds.), Academic Press, London, 1980, 121-132. MR 81m:41008

[6] T. H. Ganelius, Rational approximation in the complex plane and on the line, Ann. Acad. Sci. Fenn. Ser. A.I. 2 (1976), 129-145. MR 58:17106

[7] S.-Å. Gustafson and F. Stenger, Convergence acceleration applied to sinc approximation with application to approximation of $|x|^{\alpha}$, in: Computation and Control II(K. L. Bowers and J. Lund, eds.), Birkhäuser, Basel, 1991, 161-171. MR 92g:93006

[8] F. Keinert, Uniform approximation to $|x|^{\beta}$ by sinc functions, J. Approx. Th. 66 (1991), 44-52. MR 92d:41047

[9] M. A. Kowalski, K. A. Sikorski, and F. Stenger, Selected topics in approximation and computation, Oxford Univ. Press, Oxford, 1995. MR 97k:41001

[10] J. Lund and K. L. Bowers, Sinc methods for quadrature and differential equations, SIAM, Philadelphia, PA, 1992. MR 93i:65004

[11] M. Mori and M. Sugihara, The double exponential transformation in numerical analysis, in: Numerical Analysis in the 20th Century Vol. V: Quadrature and Orthogonal Polynomials, J. Comput. Appl. Math. 127 (2001), 287-296. MR 2001k:65041

[12] D. J. Newman, Quadrature formulae for $H^{p}$ functions, Math. Z. 166 (1979), 111-115. MR 80g:41022

[13] J. R. Rice, On the degree of convergence of nonlinear spline approximation, in: Approximations with Special Emphasis on Spline Functions (I. J. Schoenberg, ed.), Academic Press, New York, 1969, 349-365. MR 42:2226 
[14] F. Stenger, Optimal convergence of minimum norm approximations in $H_{p}$, Numer. Math. 29 (1978), 345-362. MR 58:3342

[15] F. Stenger, Numerical methods based on Whittaker cardinal or sinc functions, SIAM Rev. 23 (1981), 165-224. MR 83g:65027

[16] F. Stenger, Explicit nearly optimal linear rational approximation with preassigned poles, Math. Comput. 47 (1986), 225-252. MR 87g:41034

[17] F. Stenger, Numerical methods based on sinc and analytic functions, Springer-Verlag, New York, 1993. MR 94k:65003

[18] F. Stenger, Summary of sinc numerical methods, in: Numerical Analysis in the 20th Century Vol. I: Approximation Theory, J. Comput. Appl. Math. 121 (2000), 379-420. MR 2001d:65018

[19] M. Sugihara, Optimality of the double exponential formula - functional analysis approach, Numer. Math. 75 (1997), 379-395. MR 97i:41041

[20] H. Takahasi and M. Mori, Double exponential formulas for numerical integration, Publ. RIMS Kyoto Univ. 9 (1974), 721-741. MR 49:11781

[21] K. Wilderotter, $n$-widths of $H^{p}$-spaces in $L_{q}(-1,1)$, J. Complexity 8 (1992), 324-335. MR 94e:46051

Department of Computational Science and Engineering, Graduate School of EngiNEERING, NAGOYA University, NAGOYA 464-8603, JAPAN

E-mail address: sugihara@na.cse.nagoya-u.ac.jp 